\title{
Growth Indices as Influenced by Variety, Spacing and Nutrition in Seed Cluster Bean
}

\author{
M. Tagore Naik, D. Srihari and A.V.D. Dorajeerao* \\ Horticultural Reseach Station, Mahanandi College of Horticulture, \\ Venkataramannagudem, Andhra Pradesh, India \\ *Corresponding author
}

A B S T R A C T

\begin{tabular}{|c|c|}
\hline Keywords & \\
\hline $\begin{array}{l}\text { Growth indices, } \\
\text { Variety, Spacing, } \\
\text { Nutrition, Seed } \\
\text { guar. }\end{array}$ & \multirow{3}{*}{$\begin{array}{l}\text { The effect of planting geometry and nutrition on growth and flowering of } \\
\text { seed guar cultivars viz., HG } 365 \text { and } \mathrm{HG} 563 \text { was analysed under } \\
\text { Mahanandi conditions. The growth parameters like dry weight (g) of whole } \\
\text { plant, leaf area per plant }\left(\mathrm{cm}^{2}\right) \text {, leaf area index, crop growth rate, relative } \\
\text { growth rate and seed yield per plot }(\mathrm{kg}) \text { were recorded significantly the } \\
\text { highest values in the variety HG } 365 \text { planted at the spacing of } 30 \mathrm{~cm} \text { x } 10 \\
\mathrm{~cm} \text { and applied with the fertilizer dose of } 45 \mathrm{~N}: 60 \mathrm{P}: 60 \mathrm{~K}: 30 \mathrm{~S} \mathrm{~kg} \text { per ha. }\end{array}$} \\
\hline Article Info & \\
\hline $\begin{array}{l}\text { Accepted: } \\
\text { 28 October } 2017 \\
\text { Available Online: } \\
10 \text { December } 2017\end{array}$ & \\
\hline
\end{tabular}

\section{Introduction}

Cluster bean is botanically called as Cyamopsis tetragonoloba (L.) Taub. It belongs to the family Leguminaceae. The crop is popularly known as guar referring to its seed. India is considered as native place for guar or cluster bean. It has been used as vegetable in our country from hundreds of years. However the best spacing for seed cluster bean has not been standardized yet and therefore, it is proposed to include the factor of planting geometry levels during the designing of treatments in the present study. Further, in order to realize maximum seed yield, proper dose of nutrients is to be standardized. In the present research paper, the effect of nutritional dose, planting geometry was analysed in terms of growth indices in two seed guar varieties under Mahanandi conditions.

\section{Material and Methods}

The experiment was conducted in factorial randomized design with three factors viz., varieties (2), planting geometry levels (3) and nutritional levels (3) replicated thrice.

The plot was laid out at Horticultural Research Station, Mahanandi, Kurnool district of Andhra Pradesh during the year 2014-15 and 2015-16.

The data obtained from both the years was pooled and presented in the tables. 


\section{Results and Discussion}

\section{Dry weight of whole plant (g)}

Data on dry weight of whole plant showed non-significant differences at 30 DAS. However, the dry weight of whole plant exhibited significant variations due to variety, planting geometry, nutritional combinations as well as their interactions (Table 1) at 60 and 90 DAS. Among the varieties HG 365 recorded the highest dry weight of whole plant $(31.43 \mathrm{~g})$ at 90 DAS. Planting geometry of $30 \mathrm{~cm} \times 10 \mathrm{~cm}\left(\mathrm{~S}_{1}\right)$ recorded significantly the highest dry weight of whole plant (39.95 g) followed by $40 \mathrm{~cm} \times 10 \mathrm{~cm}\left(\mathrm{~S}_{3}\right)(29.45 \mathrm{~g})$. Application of $45 \mathrm{~N}$ : 60P: $60 \mathrm{~K}: 30 \mathrm{~S} \mathrm{~kg}$ per ha $\left(\mathrm{F}_{3}\right)$ recorded the highest dry weight of whole plant $(31.40 \mathrm{~g})$ which was on par with $30 \mathrm{~N}$ : 40P: 40K: 20S kg per ha $\left(\mathrm{F}_{2}\right)(30.85 \mathrm{~g})$. The interaction effects exhibited the similar trend as that observed in case of fresh weights.

As evident from the data on dry weight of whole plant, the photosynthetic capacity of the plant was significantly influenced by the variety, planting geometry and nutritional combinations. The fresh weight of whole plant as well as its components like leaf, stem, and pod was nothing but the carbon assimilates, synthesized by the green leaves through photosynthesis. Some of these assimilates would have been lost through respiration and the differences could be observed as the net photosynthetic product. Among the varieties, the highest values of fresh weight and dry weights at 90 DAS for whole plant as well as its components were observed in HG 365. Among the planting densities the whole plant fresh and dry weights were maximum at the widest spacing of $30 \mathrm{~cm}$ x $20 \mathrm{~cm}\left(16.7\right.$ plants per $\left.\mathrm{m}^{2}\right)$ followed by relatively closer spacing $40 \mathrm{~cm} \mathrm{x}$ $10 \mathrm{~cm}$ (25 plants per $\left.\mathrm{m}^{2}\right)$. However the fresh and dry weight of leaves exhibited minimum values with the closest spacing of $30 \mathrm{~cm} \times 10$ $\mathrm{cm}$ accommodating 33.33 plants per $\mathrm{m}^{2}$. This might be probably due to the higher number of leaves per plant in closely spaced plants as against the higher number of branches in widely spaced plants. The proportion of leaf in terms of fresh and dry weight was also found to be low with closely spaced plants as compared to widely spaced plants.

In respect of fertilizer doses the fresh and dry weight of whole plant as well as its components increased with every increase in the nutritional dose. However the increase in these weights above $F_{2}$ level was not significant with respect to leaves, indicating that additional doses above $\mathrm{F}_{2}$ level could not be productively utilised by the plant and eventually produced less leaf area and therefore could have not assimilated and diverted the assimilates in to photosynthetic organs. These results are in conformity with Naik (2007) who noticed that the whole plant dry weight was significantly superior with lower plant densities or widely spaced plants, and attributed the same due to lack of competition for growth resources during all stages of crop growth. Increase in the fresh and dry weight with higher fertiliser level was also reported by Kumavath and Khangarot (2002) and Sharma and Nehara (2004).

Prabhavathi (2005)studied dry matter accumulation among different genotypes and stated that those genotypes that had initiated bearing after 60 days from planting putforth, minimum dry matter and hence translocation of assimilates towards reproductive parts was less. Further it was noted that with increased fertiliser doses, the dry matter accumulation was found to be significantly more. It was thus inferred that, the earlier dose of nutrients had profound influence on the production of dry matter and its partitioning between the various organs of the plant, because the dry matter accumulated in the earlier stages would be directly influenced by such doses. 
Proper doses in the early stage of the crop can thus make the plant to push greater amount of assimilates to reproductive organs.

The amount of total dry matter (TDM) produced was stated as an indication of the overall efficiency of utilization of resources and better light interception. The data pertaining to total dry weight per plant in the present study indicated that, it increased continuously from 30 DAS to harvest. At later stages of crop growth, the dry matter accumulation followed decreasing trend, which was attributed to reduced source activity leading to lesser dry matter accumulation in leaf and stem. The results of the present study are in conformity with those revealed by Prabhavathi (2005).

Meena et al., (2014) also noticed significant differences among the varieties in respect of dry matter accumulation at all the growth stages. This was attributed to be due to the fast growth habit of those varieties showing high dry matter accumulation taking less time to mature. The cultivars with high dry matter accumulation took less time to mature indicating that the assimilates were compounded over time to effect maturity period and vice versa.

Ayub et al., (2011) quoted that the seeding density influenced the plant growth due to its direct relation with plant population. The higher plant population increased competition among plants for nutrients, light and space, thus reducing dry weight per plant; while lower population density caused inefficient use of natural resources and inputs (Lone et al., 2010). The dry matter yield was increased significantly with increase in nitrogen levels (Ayub et al., 2011). The highest dry matter yield was recorded with the application of top most dose of nitrogen. The increase in dry matter yield was attributed to the production of more dry matter as a result of improved photosynthetic activity at higher levels of nitrogen. Dry matter per unit area was significantly increased with increase in seeding rates or density of population. The highest dry matter yield was obtained with the highest seeding rates of $50 \mathrm{~kg} \mathrm{ha}^{-1}$.

However, these results are contradictory to those of Sheikh (2004) and Modaihsh et al., (2007) who reported that nitrogen application did not affect the dry matter accumulation. These contradictory results can be attributed to differences in climate and soil fertility.

\section{Leaf area per plant $\left(\mathrm{cm}^{2}\right)$}

Leaf area per plant varied significantly (Table 2 ) by the effect of variety, planting geometry, nutritional combinations as well as their interactions at all growth stages. Among the varieties HG 365 recorded the highest leaf area $\left(533.96 \mathrm{~cm}^{2}\right)$ at 90 DAS. Planting geometry of $30 \mathrm{~cm} \mathrm{x} 10 \mathrm{~cm}\left(\mathrm{~S}_{1}\right)$ recorded significantly the highest leaf area (509.82 $\mathrm{cm}^{2}$ ) followed by $40 \mathrm{~cm}$ x $10 \mathrm{~cm}\left(\mathrm{~S}_{3}\right)(495.30$ $\mathrm{cm}^{2}$ ). Application of $45 \mathrm{~N}: 60 \mathrm{P}: 60 \mathrm{~K}: 30 \mathrm{~S} \mathrm{~kg}$ per ha $\left(\mathrm{F}_{3}\right)$ recorded the highest leaf area $\left(536.88 \mathrm{~cm}^{2}\right)$ which was on par with $30 \mathrm{~N}$ : 40P: 40K: 20S kg per ha $\left(\mathrm{F}_{2}\right)\left(504.89 \mathrm{~cm}^{2}\right)$. Among the interactions, the two way interactions between planting geometry $v s$ nutritional combation and variety $v s$ nutritional level were found significant from the beginning i.e. 30 DAS. However, the effect of all interactions was found significant at 90 DAS.

\section{Leaf area index}

The effect of variety, planting geometry, nutritional combinations and their interactions on leaf area index (Table 3) was found to be significant at 60 and 90 DAS. However, at 30 DAS, the interaction between variety and planting geometry was non-significant during both the seasons. 
Table.1 Dry Weight (g) of whole plant as influenced by variety, planting geometry and nutritional combination (Pooled data of 2014-15 \& 2015-16)

\begin{tabular}{|c|c|c|c|c|c|c|c|c|c|c|}
\hline \multirow{3}{*}{$\begin{array}{l}\text { Planting Geometry } \\
\text { (B) }\end{array}$} & \multirow{3}{*}{$\begin{array}{l}\text { Nutritional Combination } \\
\text { (C) }\end{array}$} & \multicolumn{9}{|c|}{ Variety (A) } \\
\hline & & \multicolumn{3}{|c|}{$30 \mathrm{DAS}$} & \multicolumn{3}{|c|}{60 DAS } & \multicolumn{3}{|c|}{$90 \mathrm{DAS}$} \\
\hline & & $\begin{array}{l}\mathrm{HG} \\
365\end{array}$ & $\begin{array}{l}\mathrm{HG} \\
563\end{array}$ & Mean & $\begin{array}{l}\mathrm{HG} \\
365\end{array}$ & $\begin{array}{l}\mathrm{HG} \\
563\end{array}$ & Mean & $\begin{array}{l}\mathrm{HG} \\
365\end{array}$ & $\begin{array}{l}\mathrm{HG} \\
563\end{array}$ & Mean \\
\hline \multirow{4}{*}{$\begin{array}{l}\mathrm{S}_{1}(30 \mathrm{~cm} \times 10 \mathrm{~cm}) \\
\left(33.3 \text { plants per } \mathrm{m}^{2}\right)\end{array}$} & $\mathrm{F}_{1}(15 \mathrm{~N}: 20 \mathrm{P}: 20 \mathrm{~K}: 10 \mathrm{~S})$ & 10.56 & 8.45 & 9.51 & 22.18 & 17.75 & 19.97 & 26.62 & 21.30 & 23.96 \\
\hline & $\mathrm{F}_{2}(30 \mathrm{~N}: 40 \mathrm{P}: 40 \mathrm{~K}: 20 \mathrm{~S})$ & 10.78 & 9.17 & 9.97 & 22.64 & 19.25 & 20.95 & 27.17 & 23.10 & 25.14 \\
\hline & $\mathrm{F}_{3}(45 \mathrm{~N}: 60 \mathrm{P}: 60 \mathrm{~K}: 30 \mathrm{~S})$ & 10.97 & 9.32 & 10.15 & 23.03 & 19.58 & 21.30 & 27.64 & 23.49 & 25.57 \\
\hline & Mean & 10.77 & 8.98 & 9.88 & 22.62 & 18.86 & 20.74 & 27.14 & 22.63 & 24.89 \\
\hline \multirow{4}{*}{$\begin{array}{l}\mathrm{S}_{2}(30 \mathrm{~cm} \times 20 \mathrm{~cm}) \\
\left(16.7 \text { plants per } \mathrm{m}^{2}\right)\end{array}$} & $\mathrm{F}_{1}(15 \mathrm{~N}: 20 \mathrm{P}: 20 \mathrm{~K}: 10 \mathrm{~S})$ & 9.97 & 8.47 & 9.22 & 20.93 & 17.79 & 19.36 & 25.11 & 21.35 & 23.23 \\
\hline & $\mathrm{F}_{2}(30 \mathrm{~N}: 40 \mathrm{P}: 40 \mathrm{~K}: 20 \mathrm{~S})$ & 11.28 & 9.59 & 10.44 & 23.70 & 20.14 & 21.92 & 28.44 & 24.17 & 26.30 \\
\hline & $\mathrm{F}_{3}(45 \mathrm{~N}: 60 \mathrm{P}: 60 \mathrm{~K}: 30 \mathrm{~S})$ & 12.02 & 10.21 & 11.11 & 25.23 & 21.45 & 23.34 & 30.28 & 25.74 & 28.01 \\
\hline & Mean & 11.09 & 9.42 & 10.26 & 23.29 & 19.79 & 21.54 & 27.94 & 23.75 & 25.85 \\
\hline \multirow{4}{*}{$\begin{array}{l}\mathrm{S}_{3}(40 \mathrm{~cm} \times 10 \mathrm{~cm}) \\
\left(25 \text { plants per } \mathrm{m}^{2}\right)\end{array}$} & $\mathrm{F}_{1}(15 \mathrm{~N}: 20 \mathrm{P}: 20 \mathrm{~K}: 10 \mathrm{~S})$ & 9.72 & 8.26 & 8.99 & 20.79 & 17.67 & 19.23 & 24.50 & 20.82 & 22.66 \\
\hline & $\mathrm{F}_{2}(30 \mathrm{~N}: 40 \mathrm{P}: 40 \mathrm{~K}: 20 \mathrm{~S})$ & 11.11 & 9.44 & 10.28 & 23.77 & 20.20 & 21.98 & 28.00 & 23.80 & 25.90 \\
\hline & $\mathrm{F}_{3}(45 \mathrm{~N}: 60 \mathrm{P}: 60 \mathrm{~K}: 30 \mathrm{~S})$ & 11.21 & 9.53 & 10.37 & 23.98 & 20.39 & 22.18 & 28.25 & 24.02 & 26.14 \\
\hline & Mean & 10.68 & 9.08 & 9.88 & 22.85 & 19.42 & 21.13 & 26.92 & 22.88 & 24.90 \\
\hline \multicolumn{11}{|c|}{ For Comparing varieties (A) and Nutritional combinations (C) } \\
\hline \multirow{3}{*}{\multicolumn{2}{|c|}{$\begin{array}{l}\mathrm{F}_{1}(15 \mathrm{~N}: 20 \mathrm{P}: 20 \mathrm{~K}: 10 \mathrm{~S}) \\
\mathrm{F}_{2}(30 \mathrm{~N}: 40 \mathrm{P}: 40 \mathrm{~K}: 20 \mathrm{~S}) \\
\mathrm{F}_{3}(45 \mathrm{~N}: 60 \mathrm{P}: 60 \mathrm{~K}: 30 \mathrm{~S})\end{array}$}} & 10.08 & 8.40 & 9.24 & 21.30 & 17.74 & 19.52 & 25.41 & 21.16 & 23.28 \\
\hline & & 11.06 & 9.40 & 10.23 & 23.37 & 19.86 & 21.62 & 27.87 & 23.69 & 25.78 \\
\hline & & 11.40 & 9.69 & 10.54 & 24.08 & 20.47 & 22.28 & 28.72 & 24.42 & 26.57 \\
\hline \multicolumn{2}{|c|}{ Mean } & 10.85 & 9.16 & 10.00 & 22.92 & 19.36 & 21.14 & 27.33 & 23.09 & 25.21 \\
\hline Factor & \multicolumn{2}{|l|}{$S E m \pm$} & \multicolumn{2}{|c|}{$C D$} & \multicolumn{2}{|c|}{$S E m \pm$} & $C D$ & \multicolumn{2}{|c|}{$S E m \pm$} & $C D$ \\
\hline Variety $(A)$ & \multirow{2}{*}{\multicolumn{2}{|c|}{$\begin{array}{l}0.24 \\
0.04\end{array}$}} & \multirow{2}{*}{\multicolumn{2}{|c|}{$\begin{array}{l}0.69 \\
0.13\end{array}$}} & \multicolumn{2}{|c|}{$\begin{array}{l}0.50 \\
0.08\end{array}$} & 1.46 & \multicolumn{2}{|c|}{0.60} & 1.74 \\
\hline $\begin{array}{l}\text { Ptg. Geom. (B) } \\
\text { Nutril Combn }(C)\end{array}$ & & & & & \multicolumn{2}{|c|}{0.29} & 0.23 & \multicolumn{2}{|c|}{0.34} & $\begin{array}{l}0.32 \\
0.99\end{array}$ \\
\hline$A \times B$ & \multicolumn{2}{|l|}{-} & \multicolumn{2}{|c|}{$N S$} & \multicolumn{2}{|c|}{ - } & $\begin{array}{r}0.0 J \\
N S\end{array}$ & \multicolumn{2}{|c|}{ - } & $N S$ \\
\hline$B x C$ & \multicolumn{2}{|l|}{0.17} & \multicolumn{2}{|c|}{0.49} & 0. & & 1.01 & 0. & & 1.25 \\
\hline$A x C$ & - & & $N$ & & . & & $N S$ & . & & $N S$ \\
\hline$A \times B \times C$ & - & & $N$ & & - & & $N S$ & 1. & & 2.90 \\
\hline
\end{tabular}

$\mathrm{CD}: \mathrm{CD}$ at $5 \%$ level of significance DAS: Days after sowing 
Table.2 Leaf area per plant $\left(\mathrm{cm}^{2}\right)$ as influenced by variety, planting geometry and nutritional combination (Pooled data of 2014-15 and 2015-16)

\begin{tabular}{|c|c|c|c|c|c|c|c|c|c|c|c|}
\hline \multirow{3}{*}{ Planting Geometry (B) } & \multirow{3}{*}{\multicolumn{2}{|c|}{$\begin{array}{c}\text { Nutritional } \\
\text { Combination }(\mathrm{C})\end{array}$}} & \multicolumn{9}{|c|}{ Variety (A) } \\
\hline & & & \multicolumn{3}{|c|}{$30 \mathrm{DAS}$} & \multicolumn{3}{|c|}{$60 \mathrm{DAS}$} & \multicolumn{3}{|c|}{$90 \mathrm{DAS}$} \\
\hline & & & HG 365 & HG 563 & Mean & HG 365 & HG 563 & Mean & HG 365 & HG 563 & Mean \\
\hline \multirow{4}{*}{$\begin{array}{l}\mathrm{S}_{1}(30 \mathrm{~cm} \times 10 \mathrm{~cm}) \\
\left(33.3 \text { plants per } \mathrm{m}^{2}\right)\end{array}$} & \multirow{3}{*}{\multicolumn{2}{|c|}{$\begin{array}{l}\mathrm{F}_{1}(15 \mathrm{~N}: 20 \mathrm{P}: 20 \mathrm{~K}: 10 \mathrm{~S}) \\
\mathrm{F}_{2}(30 \mathrm{~N}: 40 \mathrm{P}: 40 \mathrm{~K}: 20 \mathrm{~S}) \\
\mathrm{F}_{3}(45 \mathrm{~N}: 60 \mathrm{P}: 60 \mathrm{~K}: 30 \mathrm{~S})\end{array}$}} & 235.87 & 200.49 & 218.18 & 514.20 & 437.07 & 475.64 & 488.49 & 415.22 & 451.85 \\
\hline & & & 270.00 & 229.50 & 249.75 & 588.59 & 500.30 & 544.45 & 559.16 & 475.29 & 517.23 \\
\hline & & & 292.52 & 248.64 & 270.58 & 637.69 & 542.04 & 589.87 & 605.81 & 514.94 & 560.37 \\
\hline & \multicolumn{2}{|c|}{ Mean } & 266.13 & 226.21 & 246.17 & 580.16 & 493.14 & 536.65 & 551.15 & 468.48 & 509.82 \\
\hline \multirow{4}{*}{$\begin{array}{l}\mathrm{S}_{2}(30 \mathrm{~cm} \times 20 \mathrm{~cm}) \\
\left(16.7 \text { plants per } \mathrm{m}^{2}\right)\end{array}$} & \multirow{3}{*}{\multicolumn{2}{|c|}{$\begin{array}{l}\mathrm{F}_{1}(15 \mathrm{~N}: 20 \mathrm{P}: 20 \mathrm{~K}: 10 \mathrm{~S}) \\
\mathrm{F}_{2}(30 \mathrm{~N}: 40 \mathrm{P}: 40 \mathrm{~K}: 20 \mathrm{~S}) \\
\mathrm{F}_{3}(45 \mathrm{~N}: 60 \mathrm{P}: 60 \mathrm{~K}: 30 \mathrm{~S})\end{array}$}} & 222.11 & 188.80 & 205.45 & 484.21 & 411.58 & 447.89 & 460.00 & 391.00 & 425.50 \\
\hline & & & 256.62 & 218.13 & 237.37 & 559.43 & 475.52 & 517.47 & 531.46 & 451.74 & 491.60 \\
\hline & & & 267.68 & 227.53 & 247.60 & 583.53 & 496.00 & 539.77 & 554.36 & 471.20 & 512.78 \\
\hline & \multicolumn{2}{|c|}{ Mean } & 248.80 & 211.48 & 230.14 & 542.39 & 461.03 & 501.71 & 515.27 & 437.98 & 476.63 \\
\hline \multirow{4}{*}{$\begin{array}{l}\mathrm{S}_{3}(40 \mathrm{~cm} \times 10 \mathrm{~cm}) \\
\left(25 \text { plants per } \mathrm{m}^{2}\right)\end{array}$} & \multirow{3}{*}{\multicolumn{2}{|c|}{$\begin{array}{l}\mathrm{F}_{1}(15 \mathrm{~N}: 20 \mathrm{P}: 20 \mathrm{~K}: 10 \mathrm{~S}) \\
\mathrm{F}_{2}(30 \mathrm{~N}: 40 \mathrm{P}: 40 \mathrm{~K}: 20 \mathrm{~S}) \\
\mathrm{F}_{3}(45 \mathrm{~N}: 60 \mathrm{P}: 60 \mathrm{~K}: 30 \mathrm{~S})\end{array}$}} & 231.03 & 196.37 & 213.70 & 503.64 & 428.09 & 465.86 & 478.46 & 406.69 & 442.57 \\
\hline & & & 264.06 & 224.45 & 244.25 & 575.65 & 489.30 & 532.48 & 546.87 & 464.84 & 505.85 \\
\hline & & & 280.58 & 238.49 & 259.53 & 611.66 & 519.91 & 565.78 & 581.07 & 493.91 & 537.49 \\
\hline & \multicolumn{2}{|c|}{ Mean } & 258.55 & 219.77 & 239.16 & 563.65 & 479.10 & 521.37 & 535.46 & 455.15 & 495.30 \\
\hline \multicolumn{12}{|c|}{ For Comparing varieties (A) and Nutritional combinations (C) } \\
\hline \multirow{3}{*}{\multicolumn{3}{|c|}{$\begin{array}{l}\mathrm{F}_{1}(15 \mathrm{~N}: 20 \mathrm{P}: 20 \mathrm{~K}: 10 \mathrm{~S}) \\
\mathrm{F}_{2}(30 \mathrm{~N}: 40 \mathrm{P}: 40 \mathrm{~K}: 20 \mathrm{~S}) \\
\mathrm{F}_{3}(45 \mathrm{~N}: 60 \mathrm{P}: 60 \mathrm{~K}: 30 \mathrm{~S})\end{array}$}} & 229.67 & 195.22 & 212.45 & 500.68 & 425.58 & 463.13 & 475.65 & 404.30 & 439.97 \\
\hline & & & 263.56 & 224.02 & 243.79 & 574.56 & 488.37 & 531.47 & 545.83 & 463.96 & 504.89 \\
\hline & & & 280.26 & 238.22 & 259.24 & 610.96 & 519.32 & 565.14 & 580.41 & 493.35 & 536.88 \\
\hline \multicolumn{2}{|c|}{ Mean } & & \multirow[t]{2}{*}{257.83} & 219.15 & 238.49 & 562.07 & 477.76 & 519.91 & 533.96 & 453.87 & 493.92 \\
\hline \multirow{2}{*}{\multicolumn{2}{|c|}{ Factor }} & S Em \pm & & \multicolumn{2}{|c|}{ CD } & S Em \pm & \multicolumn{2}{|c|}{ CD } & S Em \pm & \multicolumn{2}{|c|}{ CD } \\
\hline & & 5.47 & & \multicolumn{2}{|c|}{15.84} & 11.92 & \multicolumn{2}{|c|}{34.54} & 11.33 & 32.8 & \\
\hline Planting Geom. (B) & & 1.61 & & & & 3.50 & 10.1 & & 3.33 & 9.6 & \\
\hline Nutril. Combn.(C) & & 5.96 & & 17 & 27 & 13.35 & 38. & & 12.34 & 35. & \\
\hline$A \times B$ & & - & & $\mathbf{N}$ & & 14.65 & 42. & & 13.92 & 40. & \\
\hline $\mathbf{B} \times \mathbf{C}$ & & 7.19 & & 20 & 83 & 16.01 & 46.3 & & 14.89 & 43. & \\
\hline A $\times$ C & & 10.8 & & 31 & 46 & 24.01 & 69.5 & & 22.49 & 65. & \\
\hline$A \times B \times C$ & & - & & $\mathbf{N}$ & & 27.33 & 79.1 & & 25.65 & 74. & \\
\hline
\end{tabular}


Table.3 Leaf area index as influenced by variety, planting geometry and nutritional combination

(Pooled data of 2014-15 and 2015-16)

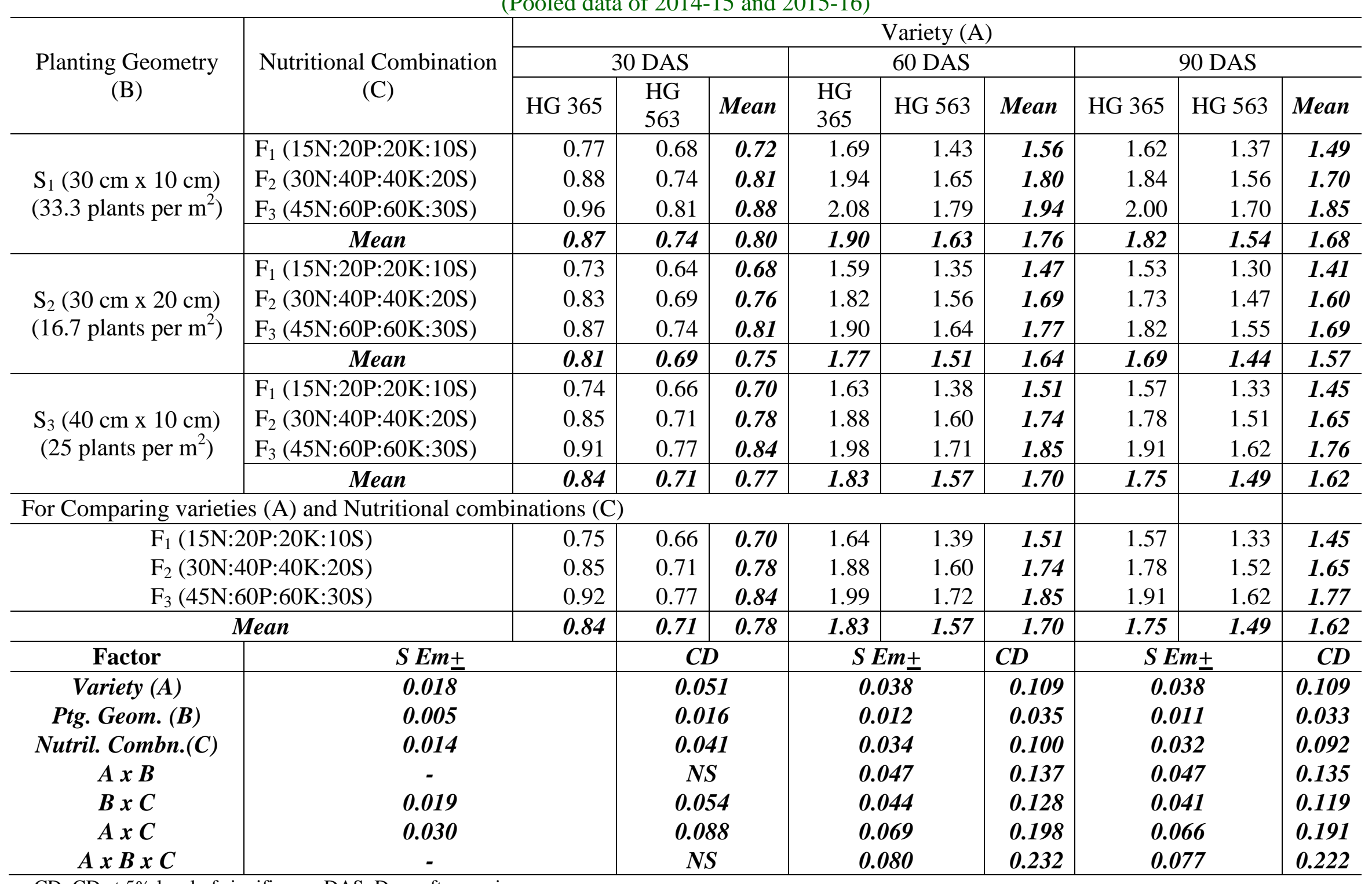

$\mathrm{CD}: \mathrm{CD}$ at $5 \%$ level of significance DAS: Days after sowing 
Table.4 Crop growth rate $\left(\mathrm{g} \mathrm{dm}^{-2} \mathrm{day}^{-1}\right)$ as influenced by variety, planting geometry and nutritional combination (Pooled data of 2014-15 and 2015-16)

\begin{tabular}{|c|c|c|c|c|c|c|c|}
\hline \multirow{3}{*}{ Planting Geometry (B) } & \multirow{3}{*}{$\begin{array}{l}\text { Nutritional Combination } \\
\text { (C) }\end{array}$} & \multicolumn{6}{|c|}{ Variety (A) } \\
\hline & & \multicolumn{3}{|c|}{$30-60$ DAS } & \multicolumn{3}{|c|}{$60-90$ DAS } \\
\hline & & HG 365 & HG 563 & Mean & HG 365 & HG 563 & Mean \\
\hline \multirow{4}{*}{$\begin{array}{l}\mathrm{S}_{1}(30 \mathrm{~cm} \times 10 \mathrm{~cm}) \\
\left(33.3 \text { plants per } \mathrm{m}^{2}\right)\end{array}$} & $\mathrm{F}_{1}(15 \mathrm{~N}: 20 \mathrm{P}: 20 \mathrm{~K}: 10 \mathrm{~S})$ & 13.69 & 12.02 & 12.85 & 7.30 & 5.69 & 6.49 \\
\hline & $\mathrm{F}_{2}(30 \mathrm{~N}: 40 \mathrm{P}: 40 \mathrm{~K}: 20 \mathrm{~S})$ & 14.68 & 12.87 & 13.77 & 8.55 & 6.73 & 7.64 \\
\hline & $\mathrm{F}_{3}(45 \mathrm{~N}: 60 \mathrm{P}: 60 \mathrm{~K}: 30 \mathrm{~S})$ & 15.17 & 13.32 & 14.25 & 8.88 & 7.00 & 7.94 \\
\hline & Mean & 14.51 & 12.74 & 13.63 & 8.25 & 6.47 & 7.36 \\
\hline \multirow{4}{*}{$\begin{array}{l}\mathrm{S}_{2}\left(30 \mathrm{~cm} \times 20 \mathrm{~cm}^{2}\right. \\
\left(16.7 \text { plants per } \mathrm{m}^{2}\right)\end{array}$} & $\mathrm{F}_{1}(15 \mathrm{~N}: 20 \mathrm{P}: 20 \mathrm{~K}: 10 \mathrm{~S})$ & 6.65 & 5.84 & 6.25 & 2.97 & 2.25 & 2.61 \\
\hline & $\mathrm{F}_{2}(30 \mathrm{~N}: 40 \mathrm{P}: 40 \mathrm{~K}: 20 \mathrm{~S})$ & 7.23 & 6.33 & 6.78 & 4.28 & 3.36 & 3.82 \\
\hline & $\mathrm{F}_{3}(45 \mathrm{~N}: 60 \mathrm{P}: 60 \mathrm{~K}: 30 \mathrm{~S})$ & 6.90 & 6.01 & 6.45 & 4.41 & 3.47 & 3.94 \\
\hline & Mean & 6.93 & 6.06 & 6.49 & 3.89 & 3.03 & 3.46 \\
\hline \multirow{4}{*}{$\begin{array}{l}\mathrm{S}_{3}(40 \mathrm{~cm} \times 10 \mathrm{~cm}) \\
\left(25 \text { plants per } \mathrm{m}^{2}\right)\end{array}$} & $\mathrm{F}_{1}(15 \mathrm{~N}: 20 \mathrm{P}: 20 \mathrm{~K}: 10 \mathrm{~S})$ & 4.92 & 4.32 & 4.62 & 2.27 & 1.73 & 2.00 \\
\hline & $\mathrm{F}_{2}(30 \mathrm{~N}: 40 \mathrm{P}: 40 \mathrm{~K}: 20 \mathrm{~S})$ & 5.55 & 4.86 & 5.20 & 3.26 & 2.56 & 2.91 \\
\hline & $\mathrm{F}_{3}(45 \mathrm{~N}: 60 \mathrm{P}: 60 \mathrm{~K}: 30 \mathrm{~S})$ & 5.57 & 4.88 & 5.22 & 3.34 & 2.63 & 2.98 \\
\hline & Mean & 5.35 & 4.68 & 5.02 & 2.95 & 2.30 & 2.63 \\
\hline \multicolumn{8}{|c|}{ For Comparing varieties $(\mathrm{A})$ and Nutritional combinations $(\mathrm{C})$} \\
\hline \multirow{3}{*}{\multicolumn{2}{|c|}{$\begin{array}{l}\mathrm{F}_{1}(15 \mathrm{~N}: 20 \mathrm{P}: 20 \mathrm{~K}: 10 \mathrm{~S}) \\
\mathrm{F}_{2}(30 \mathrm{~N}: 40 \mathrm{P}: 40 \mathrm{~K}: 20 \mathrm{~S}) \\
\mathrm{F}_{3}(45 \mathrm{~N}: 60 \mathrm{P}: 60 \mathrm{~K}: 30 \mathrm{~S})\end{array}$}} & 8.42 & 7.39 & 7.91 & 4.18 & 3.22 & 3.70 \\
\hline & & 9.15 & 8.02 & 8.59 & 5.36 & 4.21 & 4.79 \\
\hline & & 9.22 & 8.07 & 8.64 & 5.54 & 4.36 & 4.95 \\
\hline \multicolumn{2}{|c|}{ Mean } & 8.93 & 7.83 & 8.38 & 5.03 & 3.93 & 4.48 \\
\hline Factor & \multicolumn{2}{|l|}{$S E m \pm$} & \multicolumn{2}{|c|}{$C D$} & \multicolumn{2}{|c|}{$S E m \pm$} & $C D$ \\
\hline Variety $(A)$ & & \multicolumn{2}{|c|}{0.45} & \multicolumn{2}{|c|}{0.15} & 0.45 \\
\hline Ptg. Geom. (B) & \multicolumn{2}{|l|}{0.92} & \multicolumn{2}{|c|}{2.67} & \multicolumn{2}{|c|}{0.51} & 1.46 \\
\hline Nutril. Combn.(C) & \multicolumn{2}{|l|}{0.08} & \multicolumn{2}{|c|}{0.24} & \multicolumn{2}{|c|}{0.14} & 0.39 \\
\hline$A x B$ & \multicolumn{2}{|l|}{ - } & \multicolumn{2}{|c|}{$N S$} & \multicolumn{2}{|c|}{-} & $N S$ \\
\hline$B x C$ & \multicolumn{2}{|l|}{0.95} & \multicolumn{2}{|c|}{2.76} & \multicolumn{2}{|c|}{0.61} & 1.76 \\
\hline$A x C$ & \multirow{2}{*}{\multicolumn{2}{|c|}{ - }} & \multicolumn{2}{|c|}{$N S$} & . & & $N S$ \\
\hline$A x B x C$ & & & $N$ & & 0. & & 2.19 \\
\hline
\end{tabular}

$\mathrm{CD}: \mathrm{CD}$ at $5 \%$ level of significance DAS: Days after sowing 
Table.5 Relative growth rate $\left(\mathrm{mg} \mathrm{g}^{-1}\right.$ day $\left.^{-1}\right)$ as influenced by variety, planting geometry and nutritional combination (Pooled data of 2014-15 and 2015-16)

\begin{tabular}{|c|c|c|c|c|c|c|c|}
\hline \multirow{3}{*}{$\begin{array}{l}\text { Planting Geometry } \\
\text { (B) }\end{array}$} & \multirow{3}{*}{$\begin{array}{l}\text { Nutritional Combination } \\
\text { (C) }\end{array}$} & \multicolumn{6}{|c|}{ Variety $(\mathrm{A})$} \\
\hline & & \multicolumn{3}{|c|}{ 30-60 DAS } & \multicolumn{3}{|c|}{$60-90$ DAS } \\
\hline & & HG 365 & HG 563 & Mean & HG 365 & HG 563 & Mean \\
\hline \multirow{4}{*}{$\begin{array}{l}\mathrm{S}_{1}(30 \mathrm{~cm} \times 10 \mathrm{~cm}) \\
\left(33.3 \text { plants per } \mathrm{m}^{2}\right)\end{array}$} & $\mathrm{F}_{1}(15 \mathrm{~N}: 20 \mathrm{P}: 20 \mathrm{~K}: 10 \mathrm{~S})$ & 10.47 & 10.05 & 10.26 & 2.38 & 2.29 & 2.33 \\
\hline & $\mathrm{F}_{2}(30 \mathrm{~N}: 40 \mathrm{P}: 40 \mathrm{~K}: 20 \mathrm{~S})$ & 13.61 & 13.07 & 13.34 & 3.10 & 2.97 & 3.03 \\
\hline & $\mathrm{F}_{3}(45 \mathrm{~N}: 60 \mathrm{P}: 60 \mathrm{~K}: 30 \mathrm{~S})$ & 14.14 & 13.57 & 13.85 & 3.22 & 3.09 & 3.15 \\
\hline & Mean & 12.74 & 12.23 & 12.49 & 2.90 & 2.78 & 2.84 \\
\hline \multirow{4}{*}{$\begin{array}{l}\mathrm{S}_{2}(30 \mathrm{~cm} \times 20 \mathrm{~cm}) \\
\left(16.7 \text { plants per } \mathrm{m}^{2}\right)\end{array}$} & $\mathrm{F}_{1}(15 \mathrm{~N}: 20 \mathrm{P}: 20 \mathrm{~K}: 10 \mathrm{~S})$ & 10.89 & 10.46 & 10.67 & 2.48 & 2.38 & 2.43 \\
\hline & $\mathrm{F}_{2}(30 \mathrm{~N}: 40 \mathrm{P}: 40 \mathrm{~K}: 20 \mathrm{~S})$ & 14.16 & 13.59 & 13.88 & 3.22 & 3.09 & 3.16 \\
\hline & $\mathrm{F}_{3}(45 \mathrm{~N}: 60 \mathrm{P}: 60 \mathrm{~K}: 30 \mathrm{~S})$ & 14.70 & 14.11 & 14.41 & 3.34 & 3.21 & 3.28 \\
\hline & Mean & 13.25 & 12.72 & 12.99 & 3.01 & 2.89 & 2.95 \\
\hline \multirow{4}{*}{$\begin{array}{l}\mathrm{S}_{3}(40 \mathrm{~cm} \times 10 \mathrm{~cm}) \\
\left(25 \text { plants per } \mathrm{m}^{2}\right)\end{array}$} & $\mathrm{F}_{1}(15 \mathrm{~N}: 20 \mathrm{P}: 20 \mathrm{~K}: 10 \mathrm{~S})$ & 10.72 & 10.29 & 10.51 & 2.44 & 2.34 & 2.39 \\
\hline & $\mathrm{F}_{2}(30 \mathrm{~N}: 40 \mathrm{P}: 40 \mathrm{~K}: 20 \mathrm{~S})$ & 13.94 & 13.38 & 13.66 & 3.17 & 3.04 & 3.11 \\
\hline & $\mathrm{F}_{3}(45 \mathrm{~N}: 60 \mathrm{P}: 60 \mathrm{~K}: 30 \mathrm{~S})$ & 14.48 & 13.90 & 14.19 & 3.29 & 3.16 & 3.23 \\
\hline & Mean & 13.05 & 12.52 & 12.79 & 2.97 & 2.85 & 2.91 \\
\hline \multicolumn{8}{|c|}{ For Comparing varieties (A) and Nutritional combinations (C) } \\
\hline \multicolumn{2}{|c|}{$\mathrm{F}_{1}(15 \mathrm{~N}: 20 \mathrm{P}: 20 \mathrm{~K}: 10 \mathrm{~S})$} & 10.70 & 10.27 & 10.48 & 2.43 & 2.34 & 2.38 \\
\hline \multicolumn{2}{|c|}{$\mathrm{F}_{2}(30 \mathrm{~N}: 40 \mathrm{P}: 40 \mathrm{~K}: 20 \mathrm{~S})$} & 13.90 & 13.35 & 13.63 & 3.16 & 3.04 & 3.10 \\
\hline \multicolumn{2}{|c|}{$\mathrm{F}_{3}(45 \mathrm{~N}: 60 \mathrm{P}: 60 \mathrm{~K}: 30 \mathrm{~S})$} & 14.44 & 13.86 & 14.15 & 3.28 & 3.15 & 3.22 \\
\hline \multicolumn{2}{|c|}{ Mean } & 13.01 & 12.49 & 12.75 & 2.96 & 2.84 & 2.90 \\
\hline Factor & \multicolumn{2}{|l|}{ S Em \pm} & \multicolumn{2}{|c|}{ CD } & \multicolumn{2}{|c|}{ S Em \pm} & CD \\
\hline Variety (A) & \multicolumn{2}{|l|}{0.07} & & \multicolumn{2}{|c|}{0.02} & 0.05 \\
\hline Ptg. Geom. (B) & \multicolumn{2}{|l|}{0.05} & \multicolumn{2}{|c|}{0.15} & \multicolumn{2}{|c|}{0.01} & 0.03 \\
\hline Nutril. Combn.(C) & \multicolumn{2}{|l|}{0.40} & \multicolumn{2}{|c|}{1.15} & \multicolumn{2}{|c|}{0.09} & 0.27 \\
\hline $\mathbf{A} \times \mathbf{B}$ & \multicolumn{2}{|l|}{-} & \multicolumn{2}{|c|}{$\mathbf{N S}$} & \multicolumn{2}{|c|}{-} & NS \\
\hline $\mathbf{B} \times \mathbf{C}$ & \multicolumn{2}{|l|}{0.42} & \multicolumn{2}{|c|}{1.23} & \multicolumn{2}{|c|}{0.09} & 0.27 \\
\hline $\mathbf{A} \times \mathbf{C}$ & - & & NS & & & & NS \\
\hline$A \times B \times C$ & - & & NS & & & & 0.32 \\
\hline
\end{tabular}

$\mathrm{CD}: \mathrm{CD}$ at $5 \%$ level of significance DAS: Days after sowing 
Table.6 Seed yield per plot $(\mathrm{kg})$ as influenced by variety, planting geometry and nutritional combination (Pooled data of 2014-15 \& 2015-16)

\begin{tabular}{|c|c|c|c|c|c|c|c|}
\hline \multirow{3}{*}{$\begin{array}{l}\text { Planting Geometry } \\
\text { (B) }\end{array}$} & \multirow{3}{*}{$\begin{array}{c}\text { Nutritional Combination } \\
\text { (C) }\end{array}$} & \multicolumn{6}{|c|}{ Variety (A) } \\
\hline & & \multicolumn{3}{|c|}{ Kharif } & \multicolumn{3}{|c|}{ Rabi } \\
\hline & & HG 365 & HG 563 & Mean & HG 365 & HG 563 & Mean \\
\hline \multirow{4}{*}{$\begin{array}{l}\mathrm{S}_{1}(30 \mathrm{~cm} \times 10 \mathrm{~cm}) \\
\left(33.3 \text { plants per } \mathrm{m}^{2}\right)\end{array}$} & $\mathrm{F}_{1}(15 \mathrm{~N}: 20 \mathrm{P}: 20 \mathrm{~K}: 10 \mathrm{~S})$ & 2.59 & 2.31 & 2.45 & 2.31 & 2.05 & 2.18 \\
\hline & $\mathrm{F}_{2}(30 \mathrm{~N}: 40 \mathrm{P}: 40 \mathrm{~K}: 20 \mathrm{~S})$ & 2.97 & 2.64 & 2.81 & 2.64 & 2.35 & 2.50 \\
\hline & $\mathrm{F}_{3}(45 \mathrm{~N}: 60 \mathrm{P}: 60 \mathrm{~K}: 30 \mathrm{~S})$ & 3.22 & 2.86 & 3.04 & 2.86 & 2.55 & 2.71 \\
\hline & Mean & 2.93 & 2.60 & 2.77 & 2.60 & 2.32 & 2.46 \\
\hline \multirow{4}{*}{$\begin{array}{l}\mathrm{S}_{2}(30 \mathrm{~cm} \times 20 \mathrm{~cm}) \\
\left(16.7 \text { plants per } \mathrm{m}^{2}\right)\end{array}$} & $\mathrm{F}_{1}(15 \mathrm{~N}: 20 \mathrm{P}: 20 \mathrm{~K}: 10 \mathrm{~S})$ & 1.55 & 1.38 & 1.46 & 1.38 & 1.23 & 1.30 \\
\hline & $\mathrm{F}_{2}(30 \mathrm{~N}: 40 \mathrm{P}: 40 \mathrm{~K}: 20 \mathrm{~S})$ & 1.79 & 1.59 & 1.69 & 1.59 & 1.42 & 1.50 \\
\hline & $\mathrm{F}_{3}(45 \mathrm{~N}: 60 \mathrm{P}: 60 \mathrm{~K}: 30 \mathrm{~S})$ & 1.87 & 1.66 & 1.76 & 1.66 & 1.48 & 1.57 \\
\hline & Mean & 1.73 & 1.54 & 1.64 & 1.54 & 1.37 & 1.46 \\
\hline \multirow{4}{*}{$\begin{array}{c}\mathrm{S}_{3}(40 \mathrm{~cm} \times 10 \mathrm{~cm}) \\
\left(25 \text { plants per } \mathrm{m}^{2}\right)\end{array}$} & $\mathrm{F}_{1}(15 \mathrm{~N}: 20 \mathrm{P}: 20 \mathrm{~K}: 10 \mathrm{~S})$ & 2.23 & 1.99 & 2.11 & 1.99 & 1.77 & 1.88 \\
\hline & $\mathrm{F}_{2}(30 \mathrm{~N}: 40 \mathrm{P}: 40 \mathrm{~K}: 20 \mathrm{~S})$ & 2.55 & 2.27 & 2.41 & 2.27 & 2.02 & 2.14 \\
\hline & $\mathrm{F}_{3}(45 \mathrm{~N}: 60 \mathrm{P}: 60 \mathrm{~K}: 30 \mathrm{~S})$ & 2.71 & 2.41 & 2.56 & 2.41 & 2.15 & 2.28 \\
\hline & Mean & 2.50 & 2.22 & 2.36 & 2.22 & 1.98 & 2.10 \\
\hline \multicolumn{8}{|c|}{ For Comparing varieties (A) and Nutritional combinations (C) } \\
\hline \multirow{3}{*}{\multicolumn{2}{|c|}{$\begin{array}{l}\mathrm{F}_{1}(15 \mathrm{~N}: 20 \mathrm{P}: 20 \mathrm{~K}: 10 \mathrm{~S}) \\
\mathrm{F}_{2}(30 \mathrm{~N}: 40 \mathrm{P}: 40 \mathrm{~K}: 20 \mathrm{~S}) \\
\mathrm{F}_{3}(45 \mathrm{~N}: 60 \mathrm{P}: 60 \mathrm{~K}: 30 \mathrm{~S}) \\
\end{array}$}} & 2.12 & 1.89 & 2.01 & 1.89 & 1.68 & 1.79 \\
\hline & & 2.44 & 2.17 & 2.30 & 2.17 & 1.93 & 2.05 \\
\hline & & 2.60 & 2.31 & 2.45 & 2.31 & 2.06 & 2.18 \\
\hline \multicolumn{2}{|c|}{ Mean } & 2.39 & 2.12 & 2.25 & 2.12 & 1.89 & 2.01 \\
\hline Factor & \multicolumn{2}{|l|}{$S E \boldsymbol{E m} \underline{ }$} & \multicolumn{2}{|c|}{$C D$} & \multicolumn{2}{|c|}{$S E m_{\underline{ \pm}}$} & $C D$ \\
\hline Variety (A) & \multicolumn{2}{|l|}{0.04} & \multicolumn{2}{|c|}{0.11} & \multicolumn{2}{|c|}{0.03} & 0.10 \\
\hline Ptg. Geom. (B) & \multicolumn{2}{|l|}{0.11} & \multicolumn{2}{|c|}{0.33} & \multicolumn{2}{|c|}{0.10} & 0.29 \\
\hline Nutril. Combn.(C) & \multicolumn{2}{|l|}{0.06} & \multicolumn{2}{|c|}{0.16} & \multicolumn{2}{|c|}{0.05} & 0.15 \\
\hline $\mathbf{A} \times \mathbf{B}$ & \multicolumn{2}{|l|}{-} & \multicolumn{2}{|c|}{$N S$} & \multicolumn{2}{|c|}{ - } & $N S$ \\
\hline $\mathbf{B} \times \mathbf{C}$ & \multicolumn{2}{|l|}{0.16} & \multicolumn{2}{|c|}{0.47} & \multicolumn{2}{|c|}{0.14} & 0.42 \\
\hline $\mathbf{A} \times \mathbf{C}$ & \multirow{2}{*}{\multicolumn{2}{|c|}{$\overline{0.20}$}} & \multirow{2}{*}{\multicolumn{2}{|c|}{$\begin{array}{l}N S \\
0.57\end{array}$}} & & & $N S$ \\
\hline $\mathbf{A} \times \mathbf{B} \times \mathbf{C}$ & & & & & & & 0.51 \\
\hline
\end{tabular}

$\mathrm{CD}: \mathrm{CD}$ at $5 \%$ level of significance 
Among the varieties HG 365 recorded the highest leaf area index (1.75) at 90 DAS. Planting geometry of $30 \mathrm{~cm} \times 10 \mathrm{~cm}\left(\mathrm{~S}_{1}\right)$ recorded significantly the highest leaf area index (1.68) followed by $40 \mathrm{~cm}$ x $10 \mathrm{~cm}\left(\mathrm{~S}_{3}\right)$ (1.62). Application of 45N: 60P: 60K: $30 \mathrm{~S} \mathrm{~kg}$ per ha $\left(\mathrm{F}_{3}\right)$ recorded the highest leaf area index (1.77) followed by $30 \mathrm{~N}: 40 \mathrm{P}: 40 \mathrm{~K}: 20 \mathrm{~S}$ $\mathrm{kg}$ per ha $\left(\mathrm{F}_{2}\right)(1.65)$.

\section{Crop growth rate $\left(\mathrm{g} \mathrm{dm}^{-2} \mathrm{day}^{-1}\right)$}

The crop growth rate exhibited significant variations due to variety, planting geometry, nutritional combinations as well as their interactions (Table 4). The highest crop growth rate was recorded by the variety $\mathrm{HG}$ 365 (8.93) at 30 to 60 DAS. Planting geometry of $30 \mathrm{~cm} \times 10 \mathrm{~cm}\left(S_{1}\right)$ recorded significantly the highest crop growth rate (13.63) followed by $30 \mathrm{~cm}$ x $20 \mathrm{~cm}\left(S_{2}\right)$ (6.49). Application of 45N: 60P: $60 \mathrm{~K}: 30 \mathrm{~S} \mathrm{~kg}$ per ha $\left(\mathrm{F}_{3}\right)$ recorded the highest crop growth rate (8.64) which was on par with $30 \mathrm{~N}$ : $40 \mathrm{P}$ : 40K: 20S kg per ha $\left(\mathrm{F}_{2}\right)(8.59)$.

\section{Relative growth rate $\left(\mathrm{mg} \mathrm{g}^{-1} \mathrm{day}^{-1}\right)$}

The relative growth rate exhibited significant variations due to variety, planting geometry, nutritional combinations as well as their interactions (Table 5). The var. HG 365 recorded the highest relative growth rate (13.01) at 30 to 60 DAS. Planting geometry of $30 \mathrm{~cm}$ x $20 \mathrm{~cm}\left(\mathrm{~S}_{2}\right)$ recorded significantly the highest relative growth rate (12.99) followed by $40 \mathrm{~cm}$ x $10 \mathrm{~cm}\left(\mathrm{~S}_{3}\right)$ (12.79). Application of 45N: 60P: 60K: $30 \mathrm{~S} \mathrm{~kg}$ per ha $\left(\mathrm{F}_{3}\right)$ recorded the highest relative growth rate (3.22) followed by $30 \mathrm{~N}$ : 40P: 40K: $20 \mathrm{~S} \mathrm{~kg}$ per ha $\left(\mathrm{F}_{2}\right)(3.10)$.

The interaction effect was found significant between planting geometry $v s$ nutritional level at 30-60 DAS as well as 60-90 DAS. The highest RGR was recorded by the planting geometry of $30 \mathrm{~cm} \mathrm{x} 20 \mathrm{~cm}+$ nutritional level of 45N: 60P: $60 \mathrm{~K}: 30 \mathrm{~S} \mathrm{~kg}$ per ha $\left(\mathrm{F}_{3}\right)(14.41$ and 3.28, respectively at 30-60 and 60-90 DAS) followed by the planting geometry of $40 \mathrm{~cm} \times 10 \mathrm{~cm}+$ the same nutritional level (14.19 and 3.23) at 30-60 and 60-90 DAS.

\section{Seed yield per plot (kg)}

Significant differences were observed in the seed yield per plot (Table 6) due to variety, planting geometry, nutritional combinations and their interactions. Among the varieties HG 365 recorded the highest seed yield per plot $(2.39 \mathrm{~kg})$. Planting geometry of $30 \mathrm{~cm} \mathrm{x}$ $10 \mathrm{~cm}\left(S_{1}\right)$ recorded significantly the highest seed yield per plot $(2.77 \mathrm{~kg})$ followed by 40 $\mathrm{cm} \times 10 \mathrm{~cm}\left(\mathrm{~S}_{3}\right)(2.36 \mathrm{~kg})$. Application of 45N: 60P: 60K: $30 \mathrm{~S} \mathrm{~kg}$ per ha $\left(\mathrm{F}_{3}\right)$ recorded the highest seed yield per plot $(2.45 \mathrm{~kg})$ which was on par with 30N: 40P: 40K: $20 \mathrm{~S} \mathrm{~kg} \mathrm{per}$ ha $\left(F_{2}\right)(2.30 \mathrm{~kg})$. The interaction effect between planting geometry and nutritional level was found significant with respect to seed yield per plot. The highest seed yield per plot was recorded by the closest planting pattern of $30 \mathrm{~cm} \times 10 \mathrm{~cm}$ and applied with 45N: 60P: 60K: $30 \mathrm{~S} \mathrm{~kg}$ per ha (3.04) which was on par with the same planting geometry + application of $30 \mathrm{~N}$ : $40 \mathrm{P}: 40 \mathrm{~K}: 20 \mathrm{~S} \mathrm{~kg}$ per ha (2.81) and followed by the planting geometry of $40 \mathrm{~cm} \mathrm{x} 10 \mathrm{~cm}+$ application of $45 \mathrm{~N}$ : $60 \mathrm{P}$ : $60 \mathrm{~K}: 30 \mathrm{~S} \mathrm{~kg}$ per ha (2.56).

The merit of treatment combination of $30 \mathrm{~cm}$ x $10 \mathrm{~cm}+$ application of 45N: 60P: 60K: $30 \mathrm{~S}$ $\mathrm{kg}$ per ha can be attributed to be due to the corresponding superior values in terms growth indices viz., leaf area index, crop growth rate and relative growth rate.

\section{References}

Ayub, M, Khalid, M, Tariq, M., Nadeem, M. A. and Naeem, M. A. 2011. Effect of different seeding densities and nitrogen 
levels on growth, forage yield and quality attributes of cluster bean (Cyamoposis tetragonoloba Tuab.). Journal of Agricultural Technology. 7 (5): 1409-16.

Kumawat, P. D. and Khangarot, S. S. 2002. Response of sulphur, phosphorus and Rhizobium inoculation on content and uptake of nutrient of clusterbean (Cymopsis tetragonoloba L. Taub.). Environment and Ecology. 20 (4) : 80305.

Lone, B. A, Badr-ul-Hassan, Ansar-ul-Haq, S. and Khan, M. H. 2010. Effect of seed rate, row spacing and fertility levels on relative economics of soybean (Glycine $\max$ L.) under temperate conditions. African Journal of Agricultural Research.5 : 322-24.

Meena, V. K, Kaushik, M. K, Meena, R. S, Meena, V. S. and Meena, B. P. 2014. Effect of growth regulators on clusterbean (Cyamopsis tetragonoloba L.) growth under aravali hills environment in Rajasthan. Bioscan. 9 (2): 547-50.

Modaihsh, A. S, Taha, A. A. and Mahjoub, M. O. 2007. Effect of phosphorus and nitrogen fertilization and irrigation intervals on guar crop under calcarious soil. Journal of Agricultural Science. Mansoura University. 31 : 4031-39.

Naik, S. B. 2007. Effect of plant stand and phosphorus levels on productivity and quality of cluster bean (Cymopsis tetregonoloba L.). M.Sc. (Agri.) Thesis. ANGR Agricultural University, Tirupati.

Prabhavathi, V. H. 2005. Effect of plant growth regulators, organics and nutrients on growth, physiology and yield in cluster bean (Cyamopsis tetragonoloba L. Taub.). M.Sc. (Agri.) Thesis. University of Agricultural Sciences Dharwad.

Sharma, S. K. and Nehara, K. C. 2004. Effect of different varieties and fertilizer levels on yield and yield attributing characters of guar (Cymopsis tetragonoloba L. Taub). National Symposium on Arid Legumes for Sustainable Agriculture and Trade. pp 51.

Sheikh, A. A. 2004. Effect of irrigation intervals, nitrogen and phosphorus application on forage yield, carbohydrates and protein contents of guar in the central region of Saudi Arabia. Saudi Journal of Biological Science. 11 (1): 3-9.

\section{How to cite this article:}

Tagore Naik, M., D. Srihari and Dorajeerao, A.V.D. 2017. Growth Indices as Influenced by Variety, Spacing and Nutrition in Seed Cluster Bean. Int.J.Curr.Microbiol.App.Sci. 6(12): 3684-3694. doi: https://doi.org/10.20546/ijcmas.2017.612.425 